\title{
Driver's Fatigueness Estimation using Video Processing in Wavelet Transform Domain
}

\author{
Vikram Kumar ${ }^{1}$ Dr. Swati Sharma ${ }^{2}$ and Navjot Kaur ${ }^{3}$ \\ ${ }^{1,2 \text { and }}{ }^{3}$ Universal Institute of Eng. \& Tech., Lalru, Mohali Punjab, INDIA
}

\begin{abstract}
It has been observed that the fatigueless estimation is based on facial geometric features. However, the geometric features are very often prone to vary to a great extent if the facial pose changes slightly. And the results may be completely 1800 out of phase as that of the desired one. The said problem may be thought of analysis of driver's facial expression in wavelet domain using entropy of low frequency and high frequency sub band of the facial image. Further, the video movie of the driver may be decomposed into images as frames and can be analysed in wavelet domain so as to correlate the facial images to fatigueless level. In the proposed work, following objectives are proposed in order to solve the presented problem: Decomposition of video into 2d frames/images, Wavelet Decomposition of Facial images in $L L, L H$, HL and HH frequency sub-bands using haar wavelet, Entropy profile of LL and HH sub-band images and Correlation of Entropy to fatigueless Level. Keywords: Haar Wavelet,
\end{abstract}

\section{Introduction}

Driver drowsiness is considered to be an important increasing the risk of human error that could lead to cause which leads to serious traffic accidents. Safe driving is a major concern of societies all over the world. Thousands of people are killed, or seriously injured due to drivers falling asleep at the wheels each year. Recent studies show those drivers' drowsiness accounts for up to $20 \%$ of serious or fatal accidents on motorways and monotonous roads, which impair the drivers' judgment and their ability of controlling vehicles. Therefore, it is essential to develop a real-time safety system for drowsiness-related road accident prevention. Many methods have been developed and some of them are currently being used for detecting the driver's drowsiness, including the measurements of physiological features like EEG, heart rate and pulse rate, eyelid movement, gaze, head movement and behaviors of the vehicle, such as lane deviations and steering movements.

Different techniques are used in driver-fatigue monitoring systems. These techniques are divided into four categories. The first category includes intrusive techniques, which are mostly based on monitoring biomedical signals, and therefore require physical contact with the driver. The second category includes nonintrusive techniques based on visual assessment of driver's bio-behavior from face images. The third category includes methods based on driver's performance, which monitor vehicle behaviors such as moving course, steering angle, speed, braking, etc. Finally, the fourth category combines techniques from the abovementioned three categories. The computer vision based techniques from the second category are particularly effective, because the drowsiness can be detected by observing the facial features and visual biobehavior such as head position, gaze, eye openness, eyelid movements, and mouth openness.

This category encompasses a wide range of methods and algorithms with respect to data acquisition (standard, IR and stereo cameras), image processing and feature extraction (Gabor wavelets, Gaussian derivatives, Haar-like masks, etc.), and classification (neural networks, support vector machines, knowledgebased systems, etc.). The initial task in development of driver fatigue monitoring system is to build a module for reliable real-time detection and tracking of facial features such as face, eyes, mouth, gaze, and head movements.

\section{Related Works}

In the present trend suggests driving and navigation support systems are getting importance because it is crucial in supporting drivers in several conditions in automobile industry. It is important for driving support systems to detect the status/activity of driver's consciousness. Detecting onset of driver fatigue could prevent the accidents caused by drowsy driving. It is proposed to detect / analyze the driver fatigue by the application of dedicated physiological indicators such as electroencephalography and also facial features such as eyelid movements based on template matching using neural network technique for closed eyes versus opened eyes. EEG signal is one of the most predictive and reliable measurements by the analysis of alpha, beta and theta band power etc, which are considered as direct factors associated with human drowsiness. The parameters such as blink duration and opening time changes reliably with increasing drowsiness. [1]

In this paper, a vehicle driver drowsiness warning system using image processing technique with neural network is proposed. The proposed system is based on facial images analysis for warning the driver of drowsiness or inattention to prevent traffic accidents. The facial images of driver are taken by a video camera 
which is installed on the dashboard in front of the driver. A Neural network based algorithm is proposed to determine the level of fatigue by measuring the eye opening and closing, and warns the driver accordingly. The results indicated that the proposed expert system is effective for increasing safety in driving. [2]

In recent years, we have used many technologies to detect the drowsiness of a driver in the field of accident avoidance system [1]-[3]. To develop such a system we need to install some hardware components like camera inside the car, which can capture the image of the driver at a fixed interval, and an alarm system, which will alert the driver after detecting his/her level of drowsiness. Now apart from these hardware components, we need a software part also, which can detect the level of drowsiness of the driver and is the main concern of our paper. It is believed that, driver's fatigue can be easily detected by monitoring the eye status [5] [7], which is either 'open' or 'closed'. In this paper, we develop a drowsiness detection system that will accurately monitor the open or closed state of the driver's eyes in real-time. [3]

In this work various methods and algorithms for face and eyes detection are examined in order to decide which of them are applicable for use in a driver fatigue monitoring system. In the case of face detection the standard Viola-Jones face detector has shown best results, while the method of finding the eye centers by means of gradients has proven to be most appropriate in the case of eyes detection. The later method has also a potential for retrieving behavioral parameters needed for estimation of the level of driver fatigue. This possibility will be examined in future work. [4]

Driver face monitoring system is a real-time system that can detect driver fatigue and distraction using machine vision approaches. In this paper, a new approach is introduced for driver hypovigilance (fatigue and distraction) detection based on the symptoms related to face and eye regions. In this method, face template matching and horizontal projection of top-half segment of face image are used to extract hypo-vigilance symptoms from face and eye, respectively. Head rotation is a symptom to detect distraction that is extracted from face region. 确 extracted symptoms from eye region are (1) percentage of eye closure, (2) eyelid distance changes with respect to the normal eyelid distance, and (3) eye closure rate. 庺 $\square$ rst and second symptoms related to eye region are used for fatigue detection; the last one is used for distraction detection. In the proposed system, a fuzzy expert system combines the symptoms to estimate level of driver hypo-vigilance. 㕉ere are three main contributions in the introduced method: (1) simple and efficient head rotation detection based on face template matching, (2) adaptive symptom extraction from eye region without explicit eye detection, and (3) normalizing and personalizing the extracted symptoms using a short training phase. Case three contributions lead to develop an adaptive driver eye/face monitoring. Experiments show that the proposed system is relatively efficient for estimating the driver fatigue and distraction. [5]

The presented algorithm is divided into following steps:

$>$ The video movie is decomposed to 2-d image frames.

$>$ Image frames are in jpeg format being the color video.

$>$ The images are converted to gray scale image.

$>$ Haar wavelet is applied to decompose the images into LL and HH freq.sub band images.

$>$ Entropy profile of each frame is computed for the whole video.

$>$ Correlation of entropy profile with that of fatigueness and normal level is established.

$>$ The correlation is achieved by using large no. video data of normal and fatigueness at different scales.

\section{Haar Wavelet}

In this approach, the images are decomposed using the haar wavelet in four sub-bands namely LL, LH, $\mathrm{HL}$ and HH sub-bands. The LL sub-band contains the maximum energy/entropy i.e. maximum information part, however, other sub-bands contains the information about the high frequency components like edges, sharp changes etc.

For the image decomposition and feature extraction the Haar transform has been applied as a basic tool used in the wavelet transform. The method described is used for description of the whole system enabling perfect image reconstruction. The proposed algorithm of the Haar wavelet image decomposition includes image feature based segmentation and comparison of results with the watershed transform. Individual methods have been verified for simulated images and then applied for processing of selected magnetic resonance biomedical images.

All methods were designed in the Matlab environment. Wavelets are functions generated from a single function by its dilations and translations. The Haar transform forms the simplest compression process of this kind. In 1-dimension, the corresponding algorithm transforms a 2-element vector $[x(1), x(2)] \mathrm{T}$ into $[y(1), y(2)] \mathrm{T}$ by relation: 


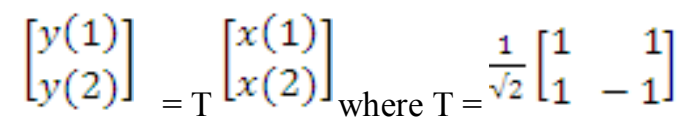

is an orthonormal matrix as its rows are orthogonal to each other (their dot products are zero). Therefore $\mathrm{T}-1=$ $\mathrm{TT}$ and it is possible to recover $\mathrm{x}$ from $\mathrm{y}$ by relation

$$
\left[\begin{array}{l}
x(1) \\
x(2)
\end{array}\right]=T^{T}\left[\begin{array}{l}
y(1) \\
y(2)
\end{array}\right]
$$

In 2-dimensions $\mathrm{x}$ and $\mathrm{y}$ become $2 \mathrm{x} 2$ matrices. We can transform at first the columns of $\mathrm{x}$, by pre-multiplying by $\mathrm{T}$, and then the rows of the result by post-multiplying by TT to find $\mathrm{y}=\mathrm{T} \mathrm{x}$ TT and in the next step $\mathrm{x}=\mathrm{TT} \mathrm{y} \mathrm{T}$

To show more clearly what is happening we can use a specific matrix $\mathrm{x}$ of the following form:

Implying that

$$
x=\left[\begin{array}{ll}
a & b \\
c & d
\end{array}\right]
$$

$$
\mathrm{y}=\frac{1}{\sqrt{2}}\left[\begin{array}{cc}
a+b+c+d & a-b+c-d \\
a+b-c-d & a-b-c+d
\end{array}\right] \quad \ldots \text { Eqn. } 1
$$

These operations correspond to the following filtering processes:

Top left: 2-D lowpass filter (Lo-Lo).

Top right: horizontal highpass and vertical low pass filter (Hi-Lo).

Lower left: horizontal lowpass and vertical high pass filter (Lo-Hi).

Lower right: 2-D highpass filter (Hi-Hi).

To apply this transform to a complete image, we group the pixels into $2 \times 2$ blocks and apply Eqn. (1) to each block.

The energies of all four sub-band images have following \%values:

$$
\begin{array}{cc}
\text { Lo }- \text { Lo } & \mathrm{Hi}-\mathrm{Lo} \\
88.2 \% & 4.0 \% \\
\text { Lo }-\mathrm{Hi} & \mathrm{Hi}-\mathrm{Hi} \\
6.3 \% & 1.5 \%
\end{array}
$$

point to the most significant compression of energy in the Lo-Lo subimage.

The wavelet coefficients are arranged as follows:

$$
\text { Coeff. }=\left[\begin{array}{cc}
c_{a} & c_{h} \\
c_{v} & c_{d}
\end{array}\right]
$$

Flow Chart






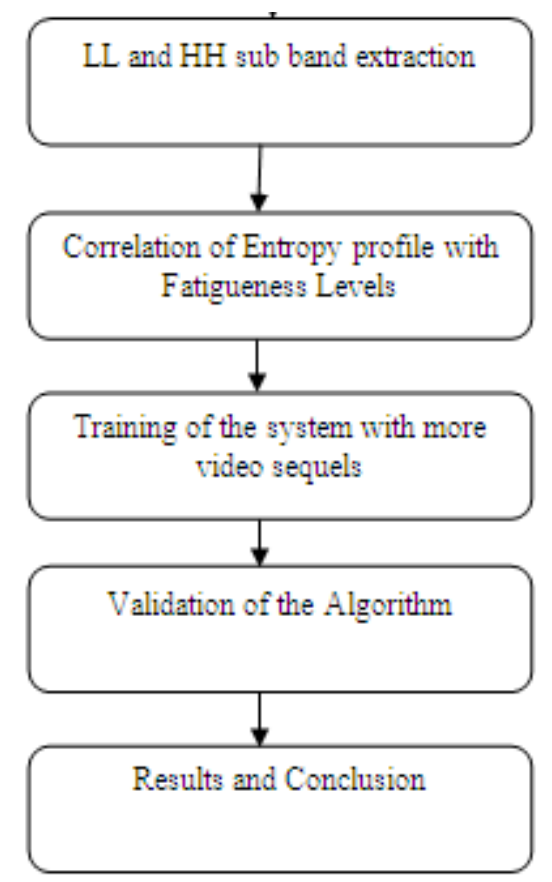

\section{Results}

Driver Fatigueness Level Analysis Data

Date: 23- 8-2014

Time: $16-18-16$

File Path $=$ D: $\backslash \backslash$ Program $\backslash$ Results.TXT

No. of Events $=30$

Threshold Activity $=3.000000$

Total Monitor Time $\quad=60$ secs.

Total Monitoring Interval $\quad=2$ secs.

Mean Entropy (Gray) over time 60 secs $=6.64$

Mean Entropy (BW ) over time 60 secs $=0.95$

Eye Open Time over time 60 secs $=30$ secs.

Eye close Time over time 60 secs $=0$ secs.

Yawnng Effect over time 60 secs $=0.407654$

ActiveStatus $=\mathrm{f}\left(\mathrm{E} \_\right.$gray, $\mathrm{E} \_$bw, ECT, EOT, Yawning $)$

ActiveStatus $=$ K1.E_gray + K2.E_bw + K3.ECT + K4.EOT + K5.Yawning

ActiveStatus $=(\mathrm{K} 1 * 6.64)+(\mathrm{K} 2 * 0.95)+(\mathrm{K} 3 * 30.00)+$

$(\mathrm{K} 4 * 0.00)+(\mathrm{K} 5 * 0.41)=38.00$



Fig. 1 Snapshot of the Program 


\section{Conclusion}

The proposed algorithm has been tested on personal car driver for testing purposes. For authentic results, the camera position has to be focused on the driver's face. Further, the algorithm has been tested in day time driving. The results suffer in night car driving as the light from the following vehicles degrades the picture quality due to reflection.

\section{References}

[1]. G.N. Keshava 1,2 Murthy and 2Zaved Ahmed Khan, "Smart Alert System for Driver Drowsiness Using EEG and Eyelid Movements", Middle-East Journal of Scientific Research 14 (5): 610-619, 2013

[2]. Itenderpal singh1, Prof. V.K.Banga, "DEVELOPMENT OF A DROWSINESS WARNING SYSTEM USING NEURAL NETWORK", International Journal of Advanced Research in Electrical, Electronics and Instrumentation Engineering (An ISO 3297: 2007 Certified Organization) Vol. 2, Issue 8, August 2013

[3]. Mr. Susanta Podder1 Mrs. Sunita Roy2, "Driver's drowsiness detection using eye status to improve the road safety", International Journal of Innovative Research in Computer and Communication Engineering (An ISO 3297: 2007 Certified Organization) Vol. 1, Issue 7, September 2013

[4]. Markan Lopar and Slobodan Ribarić, “An Overview and Evaluation of Various Face and Eyes Detection Algorithms for Driver Fatigue Monitoring Systems", Proceedings of the Croatian Computer Vision Workshop, Year 1 September 19, 2013, Zagreb, Croatia

[5]. Mohamad-Hoseyn Sigari,1 Mahmood Fathy,2 and Mohsen Soryani, “A Driver Face Monitoring System for Fatigue and Distraction Detection”, International Journal of Vehicular Technology Volume 2013, Article ID 263983, 11 pages

\section{Author Profile}

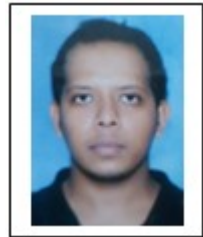

The author ${ }^{1}$ is pursuing his M.Tech. in ECE from UIET, Lalru, Mohali. His field of interest is in digital image and signal processing based system integrations and implementations. 